\title{
Human Pathogenic Candida Species Respond Distinctively to Lactic Acid Stress
}

\author{
Isabella Zangl ${ }^{1}$, Reinhard Beyer ${ }^{1}$, Ildiko-Julia Pap ${ }^{2}$, Joseph Strauss ${ }^{1} \mathbb{D}$, Christoph Aspöck ${ }^{2}$, \\ Birgit Willinger ${ }^{3}(1)$ and Christoph Schüller $1,4, *$ (D) \\ 1 Department of Applied Genetics and Cell Biology (DAGZ), Institute of Microbial Genetics, \\ University of Natural Resources and Life Sciences, 3430 Vienna (BOKU), Austria; \\ isabella.zangl@boku.ac.at (I.Z.); reinhard.beyer@boku.ac.at (R.B.); joseph.strauss@boku.ac.at (J.S.) \\ 2 Institute for Hygiene and Microbiology, University Hospital of St. Pölten, Dunant-Platz 1, 3100 St Pölten, Austria; \\ Ildiko-Julia.Pap@stpoelten.lknoe.at (I.-J.P.); Christoph.Aspoeck@stpoelten.lknoe.at (C.A.) \\ 3 Division of Clinical Microbiology, Department of Laboratory Medicine, Medical University of Vienna, \\ 1090 Vienna, Austria; birgit.willinger@meduniwien.ac.at \\ 4 Bioactive Microbial Metabolites (BiMM), Department of Applied Genetics and Cell Biology (DAGZ), \\ Institute of Microbial Genetics, University of Natural Resources and Life Sciences, 3430 Vienna, Austria \\ * Correspondence: christoph.schueller@boku.ac.at; Tel.: +43-1-47654-94484
}

Received: 12 November 2020; Accepted: 5 December 2020; Published: 8 December 2020

check for updates

\begin{abstract}
Several Candida species are opportunistic human fungal pathogens and thrive in various environmental niches in and on the human body. In this study we focus on the conditions of the vaginal tract, which is acidic, hypoxic, glucose-deprived, and contains lactic acid. We quantitatively analyze the lactic acid tolerance in glucose-rich and glucose-deprived environment of five Candida species: Candida albicans, Candida glabrata, Candida parapsilosis, Candida krusei and Candida tropicalis. To characterize the phenotypic space, we analyzed 40-100 clinical isolates of each species. Each Candida species had a very distinct response pattern to lactic acid stress and characteristic phenotypic variability. C. glabrata and C. parapsilosis were best to withstand high concentrations of lactic acid with glucose as carbon source. A glucose-deprived environment induced lactic acid stress tolerance in all species. With lactate as carbon source the growth rate of C. krusei is even higher compared to glucose, whereas the other species grow slower. C. krusei may use lactic acid as carbon source in the vaginal tract. Stress resistance variability was highest among C. parapsilosis strains. In conclusion, each Candida spp. is adapted differently to cope with lactic acid stress and resistant to physiological concentrations.
\end{abstract}

Keywords: lactic acid tolerance; Candida; candidiasis; phenotypic variability

\section{Introduction}

The human body hosts complex microbial communities [1,2]. Several Candida spp. such as $C$. albicans and C. glabrata are common human commensals, and are thus highly adapted to humans [3]. They can be found on human skin and mucous membranes like oral or vaginal epithelium and urogenital tract $[4,5]$. These fungi are opportunistic pathogens, causing mild infections like vulvovaginal candidiasis (VVC) or oral thrush [6], as well as more severe systemic infections in immunocompromised patients. Around $50 \%$ of infections are caused by C. albicans [7]. Second most common cause is C. glabrata with $15-25 \%$ of all infections [7-9]. Other relevant pathogenic strains are C. tropicalis, C. krusei, and $C$. parapsilosis $[10,11]$. Recently, $C$. auris an emerging highly drug resistant species became a focus of concern [12].

Candida species are adapted to thrive in the various environmental niches of the human body [13]. Nutrient availabilty, $\mathrm{pH}$ fluctuations, and oxygen supply vary greatly between different parts in 
the human body. For example, $\mathrm{pH}$ can be slightly alkaline (pH 7.4) in blood and tissue to acidic $(\mathrm{pH} 2-\mathrm{pH} 6)$ in gastrointestinal and vaginal tract. In this study we focus on conditions of the vaginal tract, as about $75 \%$ of all women suffer of a vaginal Candida infection at least once during a liftetime [14]. The environment is glucose deprived, possesses a relatively low $\mathrm{pH}(\mathrm{pH} 4 \pm 0.5)$ accompanied with a up to $110 \mathrm{mM}$ lactic acid and hypoxia [15]. Additionally, it is subjected to regular and developmental environmental changes (e.g., $\mathrm{pH}$ rises during menstruation). The vaginal microbiota is dominated by Lactobacillus spp., with microbiomes consisting of L. crispatus, L. jensenii, L. gasseri, and/or L. iners being the most prominent ones [16,17]. Vaginal lactic acid stems mainly from Lactobacillus spp., as human epithelial cells only produce L-lactic acid and the lactic acid in the vaginal tract consists of more than $50 \%$ D-lactic acid [18]. However, lactic acid composition and $\mathrm{pH}$ slighly depends on the dominating Lactobacillus spp. as L. crispatus dominated microbiome is generally associated with lower $\mathrm{pH}$ and a higher amount of D-lactic acid compared to a L. iners microbiome [15,19]. Vaginal Lactobacillus species generally differ in their ability to produce lactic acid isomers with $L$. iners only producing L-lactic acid, whereas L. jensenii only produces D-lactic acid [19].

Response mechanisms to acid stress differ between Candida species. In C. glabrata lactic acid response was linked to the hyperosmolarity glycerol response (HOG) pathway and deletion of Hog1 was found to be crucial for tolerance of lactic acid at physiological levels [20]. In glucose-rich environments, C. albicans stress response to weak organic acids like lactic acid or acetic acid was reported to be dependent on the transcription factor CaMig1 [21]. Acid stress response mechanisms in other Candida species have yet to be investigated.

The role of lactic acid as an antifungal agent is under debate. Lactic acid was reported to effectively reduce $C$. albicans growth [22]. However, a recent report claimed, that physiological concentrations of lactic acid at low $\mathrm{pH}$ do not reduce growth of $C$. albicans and C. glabrata [23]. Lack of consensus in this point originates possibly also from different growth medium and the used strains. Lactic acid concentration might reach levels higher than $110 \mathrm{mM}$ in vaginal micro milieu or close to a Lactobacillus biofilm. Lactobacillus spp. are reported to be viable at concentrations of up to $1 \mathrm{M}$ lactic acid at $\mathrm{pH}$ 4.5 [24]. The influence of lactic acid against Candida species has not been systematically explored yet.

We report here a systematic and quantitative analysis of the effect of lactic acid at low $\mathrm{pH}$ on relevant Candida species. To avoid phenotypic strain bias, we analyzed for each species small populations of 40 to 100 clinical isolates. We found distinct differences between and within the species populations. Furthermore, since glucose is limited in the vaginal tract, we evaluated also the lactic acid stress response in medium with a nonfermentable carbon source. Additionally, we investigated how efficient different Candida species utilize lactic acid at environmental $\mathrm{pH}$ as carbon source and found C. krusei to deviate from the other species.

\section{Materials and Methods}

\subsection{Microbial Strains}

The clinical Candida isolates were collected and provided by the Institute of Hygiene and Microbiology at University Hospital St. Pölten and the Department of Laboratory Medicine at General Hospital Vienna. Isolates were identified by MS, Chromagar Candida (BBL ${ }^{\mathrm{TM}}$ CHROMagar ${ }^{\mathrm{TM}}$ Candida Medium, Becton Dickinson GmbH, Heidelberg, Germany) and finally through DNA sequence analysis of the ITS region. A list of the strain's designations can be found in the Supplementary Table S1 [25].

\subsection{Lactate Growth Assay}

YNB medium (BD Difco ${ }^{\mathrm{TM}}$ Yeast Nitrogen Base without Amino Acids, FisherScientific, Loughborough, UK), supplemented with ammonium sulphate was prepared with $2 \%(w / v)$ L-lactate (Carl Roth $\mathrm{GmbH}$, Karlsruhe, Germany) or $2 \%(w / v)$ glucose. $\mathrm{pH}$ was adjusted to $\mathrm{pH} 4$ with $\mathrm{HCl}$. Candida isolates were grown overnight at $37{ }^{\circ} \mathrm{C}$ in YPD (1\% yeast extract, $2 \%$ peptone, $2 \%$ glucose) media in a 96-well flat bottom plate. Using a Hamilton Starlet8 robot (Hamilton Bonaduz AG, Bonaduz, 
Switzerland) each culture was diluted 1:100 into the respective medium and incubated at $37^{\circ} \mathrm{C}$. Incubation was done in at least triplicates. $\mathrm{OD}_{600 \mathrm{~nm}}$ was measured using a fully automated set-up (Cytomat42, Thermo Fisher Scientific, Waltham, MA, USA; Synergy H1 reader, BioTek Instruments Inc., Winooski, VT, USA; Rack Runner 720, Hamilton Bonaduz AG, Bonaduz, Switzerland). Raw data can be found in Supplementary Table S3.

\subsection{Lactic Acid Stress Resistance}

Isolates were grown overnight on YPD agar in CELLSTAR ${ }^{\circledR}$ OneWell Plate (Greiner Bio-One

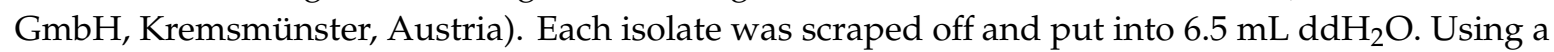
Hamilton Starlet8 robot (Hamilton Bonaduz AG, Bonaduz, Switzerland), each isolate was inoculated 1:10 diluted into $200 \mu \mathrm{L}$ medium per well of standard 96-well flat bottom plates containing a series of L-lactic acid concentrations. Inoculum for each sample was adjusted to $\mathrm{OD}_{600 \mathrm{~nm}} \sim 0.04$. YP media was prepared with $2 \%$ glucose or $2 \%$ glycerol (Carl Roth GmbH, Karlsruhe, Germany) as carbon source. Media was supplemented with 80\% L-lactic acid solution (Carl Roth GmbH, Karlsruhe, Germany) to achieve the following end concentrations: $160 \mathrm{mM}, 320 \mathrm{mM}, 480 \mathrm{mM}, 640 \mathrm{mM}$, and $800 \mathrm{mM}$ and $\mathrm{pH}$ was adjusted to $\mathrm{pH} 4$. Plates were incubated at $37^{\circ} \mathrm{C}$ for $65 \mathrm{~h}$. $\mathrm{OD}_{600 \mathrm{~nm}}$ was measured every $2 \mathrm{~h}$ using our fully automated set-up. Raw data can be found in Supplementary (Glucose-Table S2; Glycerol-Table S4).

\subsection{Statistical Analysis}

All experiments were performed at least in triplicates. All growth rates were calculated using the "growthcurver" package of the statistic software R [26,27]. Graphs were prepared using packages "ggplot2" and "ggstatsplot" $[28,29]$. Significance testing for L-lactic acid stress experiments were performed using the "ggstatsplot" package. Pairwise comparisons were done using a Games-Howell test (Welch's ANOVA) with Bejamini and Hochberg method for $p$-value adjustment. Significance testing for the cluster analysis was done using Welch's $t$-test.

\section{Results}

\subsection{Candida Species Have a Distinct Growth Rate Response to Lactic Acid Stress}

In the vaginal tract, Candida species are confronted with an overall lactic acid concentration of 110 $\mathrm{mM}$ at $\mathrm{pH} 4$ [15]. This concentration could be potentially higher in close proximity to the epithelium cell wall, as it is layered by Lactobacillus spp., the main producers of vaginal lactic acid and free diffusion might be reduced by the viscosity of the vaginal fluids and the biofilm. To assess the fitness of different Candida species against lactic acid stress, we tested populations of clinical isolates of the main Candida species found in the vaginal tract: C. albicans, C. glabrata, C. krusei, C. parapsilosis, and C. tropicalis [30]. Growth fitness tests were performed by continuous observation of the optical density. Thus, we were able to extract all parameters of the growth curves and use them for analysis of growth parameters. We used several isolates for each species to obtain data on the phenotypic variability. The different responses to lactic acid depending on the Candida species are shown in Figure 1. As expected, none of the tested species was inhibited by lactic acid concentrations close to physiological concentration in the vaginal tract, but their growth rate was reduced significantly (Figure 1). The pattern of $C$. krusei isolates represented an exception, as they grew significantly faster at $160 \mathrm{mM}$ lactic acid compared to control with no lactic acid (Figure 1C). Interestingly, growth performance at low lactic acid concentration was generally not indicative for the performance at higher concentrations. For example, C. krusei isolates were the most affected strains at $640 \mathrm{mM}$ and $800 \mathrm{mM}$ lactic acid, despite increase in growth rate at $160 \mathrm{mM}$ (Figure 1C). C. tropicalis isolates showed high reduction (about $40 \%$ ) of average growth rate of all species at $160 \mathrm{mM}$ compared to $0 \mathrm{mM}$ lactic acid control but no significant change between $160 \mathrm{mM}$ up to $480 \mathrm{mM}$ lactic acid (Figure 1E). We observed that C. albicans isolates had the most uniform reduction of growth rate throughout the different lactic acid concentration tested (Figure 1A). 

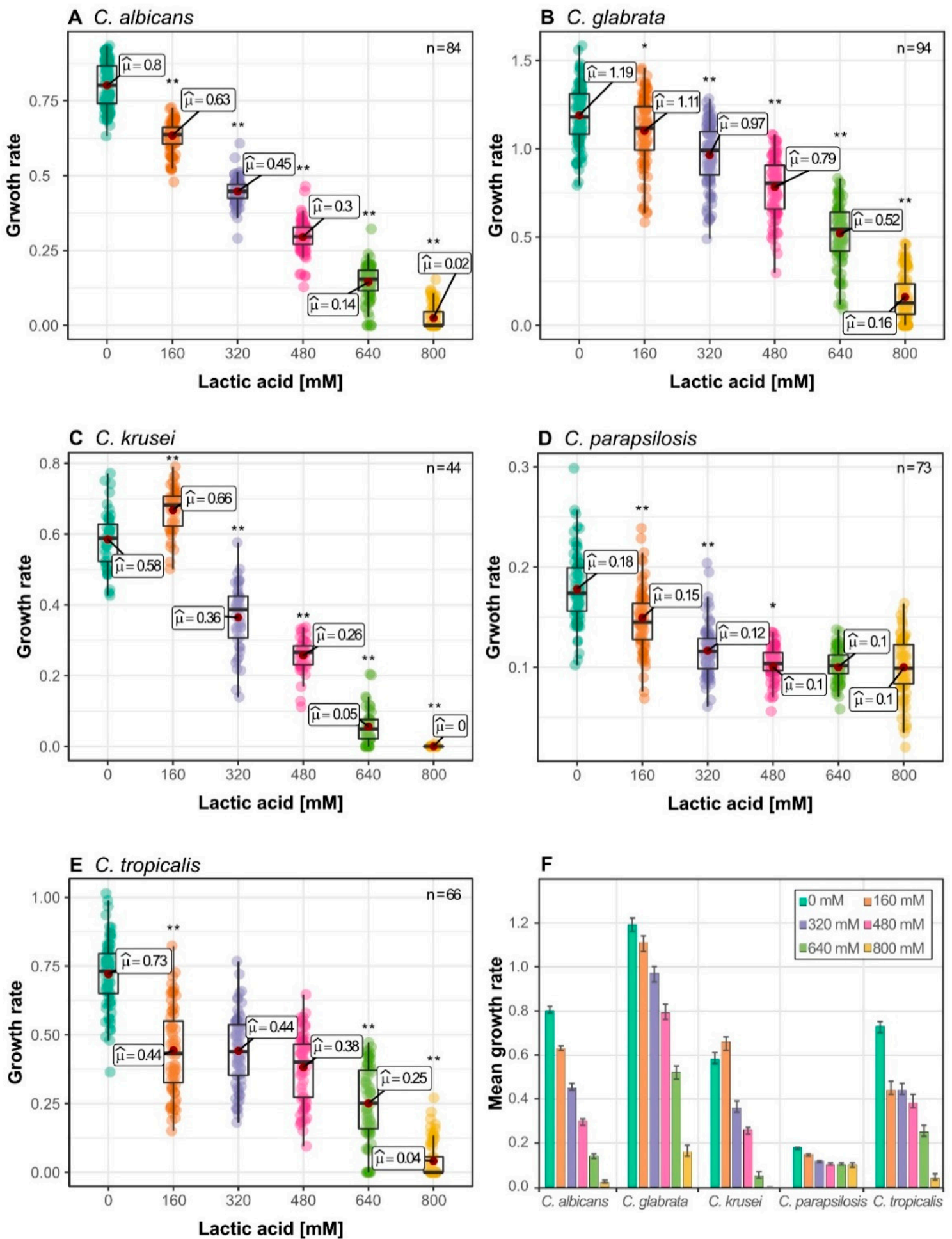

Figure 1. Growth of different Candida species under lactic acid stress with $2 \%$ Glucose as carbon source: (A) C. albicans; (B) C. glabrata; (C) C. krusei; (D) C. parapsilosis; (E) C. tropicalis; (F) Mean growth rates of different Candida species to lactic acid stress, Error bars represent confidence interval of 95\%; Each data point represents mean growth rate of triplicates measurements; Asterisks represents statistical difference of one condition to the next lower lactic acid concentration $\left({ }^{*} p \leq 0.05,{ }^{* *} p \leq 0.001\right)$. Raw data can be found in Supplementary Table S2.

C. albicans was found to be highly susceptible to higher concentrations, experiencing a reduction of growth rate of already $62 \%$ at $480 \mathrm{mM}$ (Figure 1A,F). C. glabrata isolates showed the highest population 
variability (Figure 1B), indicating different clusters of strains with specific responses. It was by far the fasted growing Candida species, with approximately $50 \%$ of all isolates growing faster than all isolates of other species at a concentration of $800 \mathrm{mM}$ (Figure 1B). C. parapsilosis was the slowest growing species, but possessed the highest resistance against lactic acid stress with the average growth rate of the population only reduced by $45 \%$ at $800 \mathrm{mM}$ lactic acid (Figure 1D). Additionally, the C. parapsilosis population appeared to have increase variability at $800 \mathrm{mM}$ lactic acid, whereas other species lose variability at higher concentrations.

\subsection{Isolates form Clusters with Similar Growth Pattern within Species}

Isolates of the same species do not always behave uniformly under environmental influences. To find clusters of different growth behaviour, we analysed the isolates in the upper and lower whiskers at $160 \mathrm{mM}$, except for C. parapsilosis where we grouped the isolates according to growth rate at $800 \mathrm{mM}$ (Figure 2). We expected to see clusters of good performing and bad performing isolates. However, only C. glabrata isolates followed this trend (Figure 2B).

Interestingly, C. krusei and C. tropicalis isolates which had a reduced growth rate at $160 \mathrm{mM}$, where faster at higher lactic acid concentrations when compared to the faster growing isolates at $160 \mathrm{mM}$ (Figure 2C,E). Some C. parapsilosis isolates were fast growing at $800 \mathrm{mM}$ lactic acid but only average at lower lactic acid concentration (Figure 2D). C. albicans was the only species without distinct clusters (Figure 2A). We also tested grouping of the isolates according to different lactic acid concentration, with essentially similar results. This is interesting since the genetic difference between C. parapsilosis strains is comparably low compared to the $C$. albicans isolates. Therefore, we can conclude that at higher lactic acid concentrations minute epigenetic differences lead to variable phenotypes.

\subsection{Candida krusei Utilizes Lactate More Efficiently Than Glucose}

In the vaginal tract, glucose is not directly available as carbon source for Candida species, however, lactic acid is found at a constant concentration. We monitored the growth of Candida species on lactate at $\mathrm{pH} 4$, to quantify their ability to utilize lactate at a $\mathrm{pH}$ similar to the one in the vaginal tract (Figure 3 and Supplementary Figure S1).

Overall, Candida species grow significantly slower on lactate than on glucose with the stark exception of C. krusei. C. krusei isolates had a significantly higher growth rate with lactate compared to glucose. When compared to their respective growth on glucose, C. parapsilosis and C. tropicalis were found to only have a growth rate reduction at about $50 \%$, making them fairly good lactate utilizer. Despite C. glabrata being the fastest growing species on glucose, it had the highest reduction of growth rate on lactate. Whereas growth on glucose shows the isolates had a high variability of maximal growth rate with glucose as corbon source, the differences diminished when grown on lactate. Similar results were obtained with the C. albicans isolates tested. Overall, the investigated Candida species vary dramatically in their ability to utilize lactate and except for $C$. krusei they are able to utilize glucose more efficiently. 

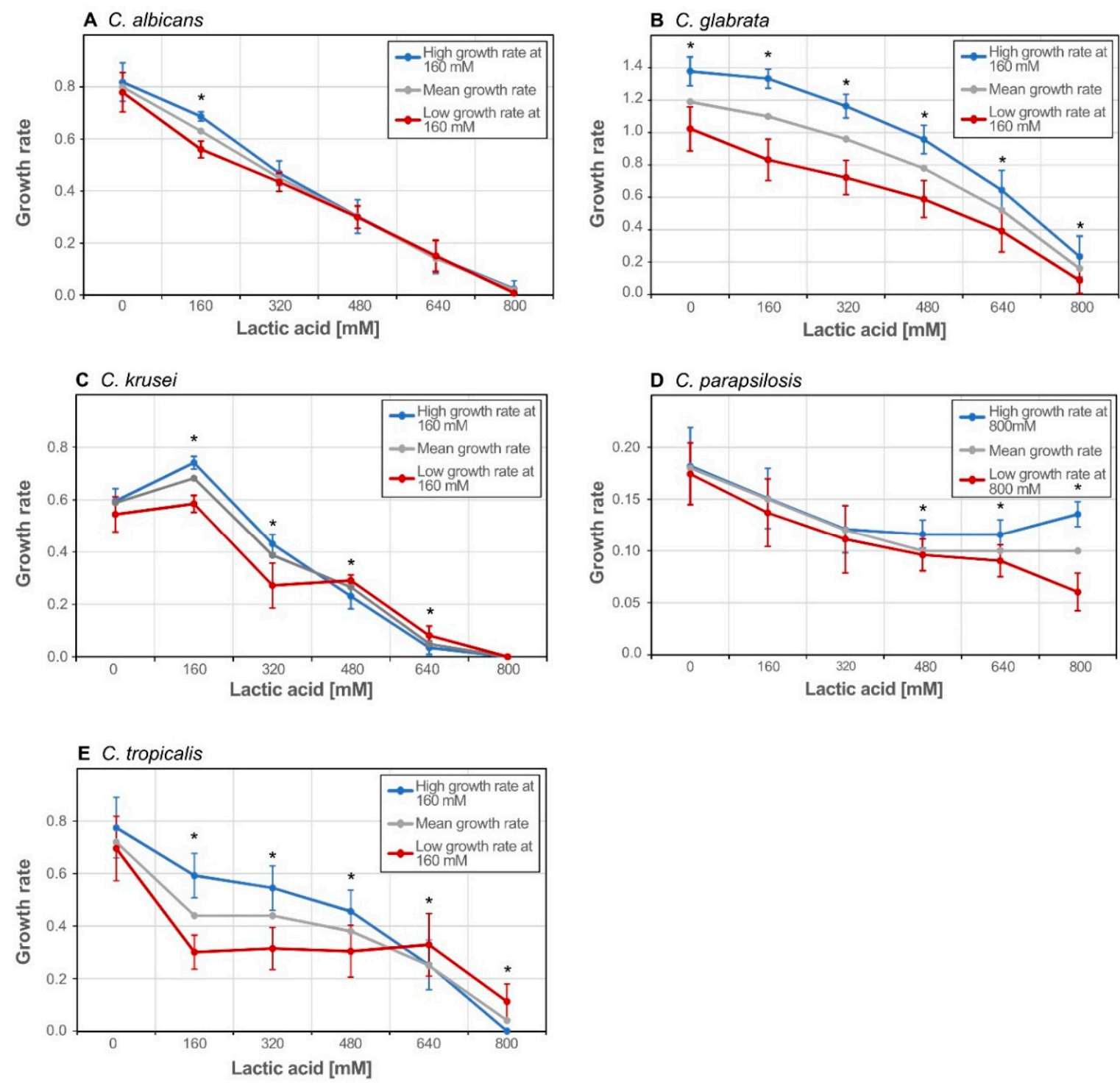

Figure 2. Cluster analysis of lactic acid stress response on Glucose. Isolates were sorted according to their growth rate at $160 \mathrm{mM}$ lactic acid if not stated otherwise and put into high (blue line) and low (red line) growth rate clusters; (A) C. albicans; (B) C. glabrata, (C) C. krusei; (D) C. parapsilosis, sorted according to the growth rates at $800 \mathrm{mM}$; (E) C. tropicalis; Grey line represents the average growth rate per concentration; Error bars represent the standard deviation of the growth rate in a cluster. Asterisks represents statistical difference of one condition to the next lower lactic acid concentration $\left({ }^{*} p \leq 0.05\right)$. 


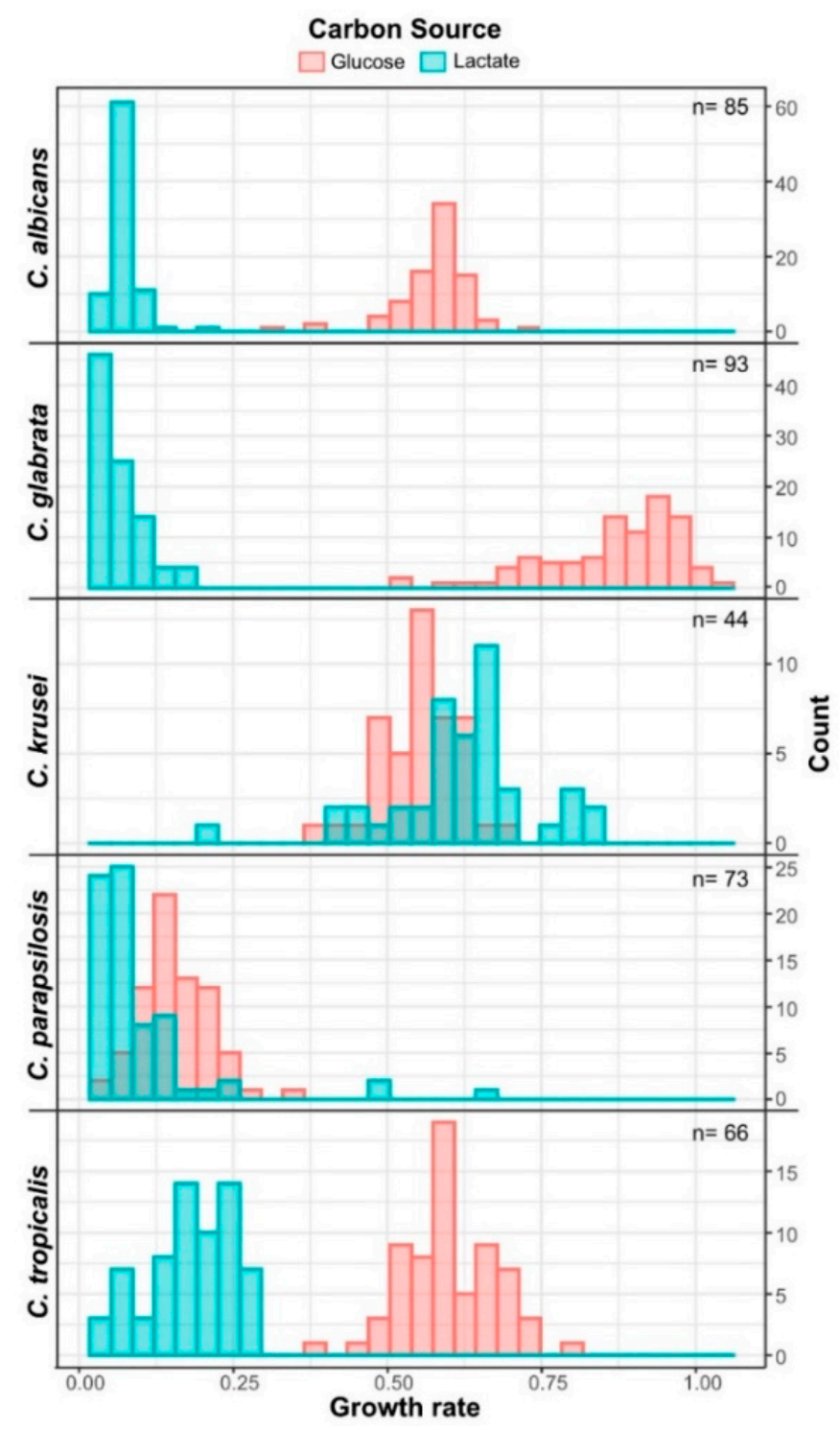

Figure 3. Distribution of growth rate of Candida isolates on $2 \%$ Lactate or $2 \%$ Glucose as sole carbon source at $\mathrm{pH} 4$; Experiment was done in at least triplicates; Mean values of maximal growth rates are reported. Raw data can be found in Supplementary Table S3.

\subsection{Lactic Acid Response Differs between Glucose-Limited and Glucose-Rich Conditions}

Finally, we analysed lactic acid stress in glucose limited conditions to approach the interplay between carbon source and lactic acid stress. We used glycerol as carbon source, as glycerol is naturally present in the vaginal tract [31]. Interestingly, growth rate of all tested Candida species was not significantly influenced by $160 \mathrm{mM}$ lactic acid compared to control (Figure 4). C. krusei was the fastest growing species (Figure $4 \mathrm{~F}$ ) and again, displayed a higher growth rate at $160 \mathrm{mM}$, compared to $0 \mathrm{mM}$ (Figure 4C). Interestingly, C. krusei average growth rate was reduced by $77 \%$ at $320 \mathrm{mM}$, representing a similar low growth rate compared to other species (Figure $4 \mathrm{~F}$ ). With reduction of growth, the population variability of C. krusei was also reduced at $320 \mathrm{mM}$ lactic acid (Figure $4 \mathrm{~F}$ ). C. albicans growth rate, which on glucose is effectively inhibited by lactic acid, was similar up to $640 \mathrm{mM}$ (Figure 4A). C. glabrata (Figure 4B) and C. tropicalis (Figure 4E) had no significant change in growth rate up to $480 \mathrm{mM}$ lactic acid. Surprisingly, no significant growth rate reduction was observed for C. aparapsilosis (Figure 4D), making it again the most lactic acid tolerant species. All in all, these results show an increased tolerance to lactic acid when glycerol is present as carbon source. 
A C. albicans

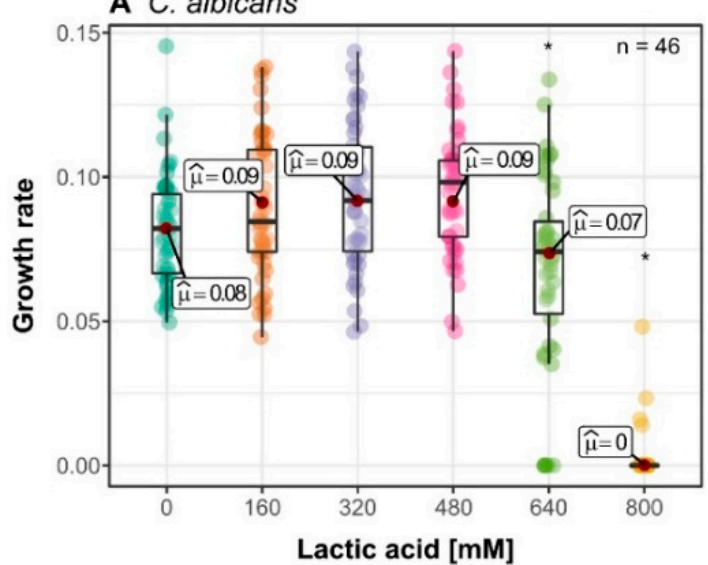

C C. krusei

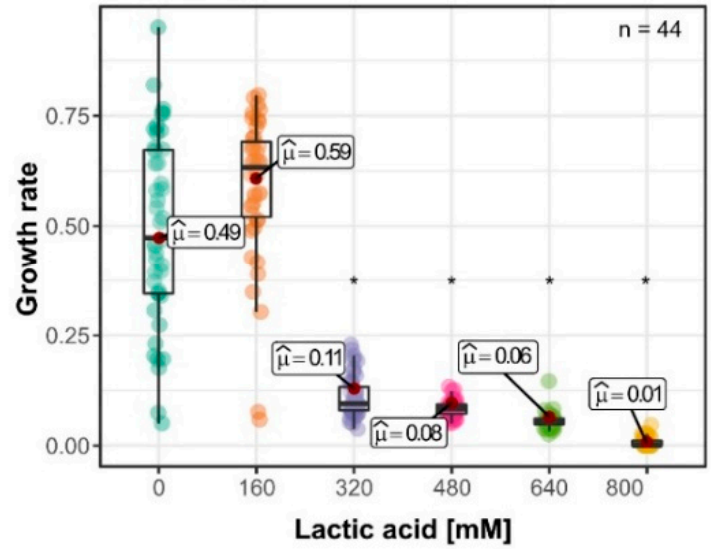

E C. tropicalis

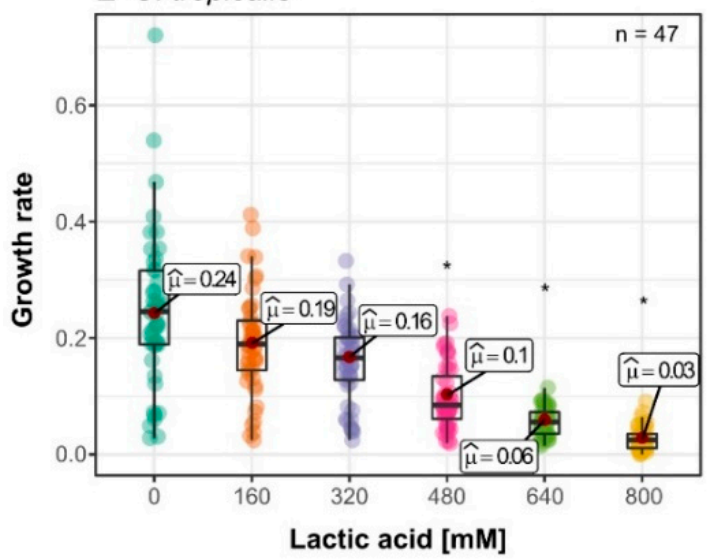

B C. glabrata

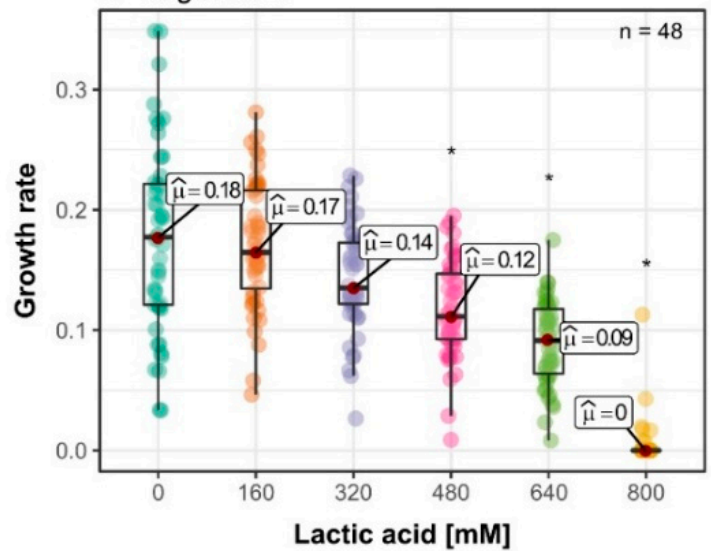

D C. parapsilosis

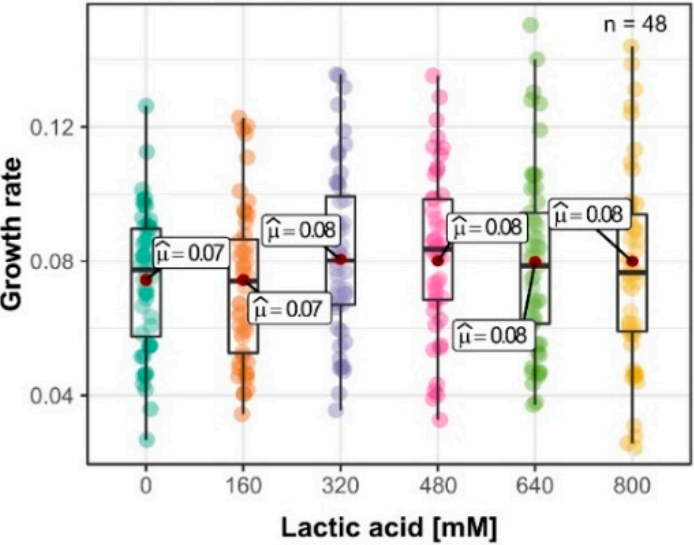

$\mathbf{F}$

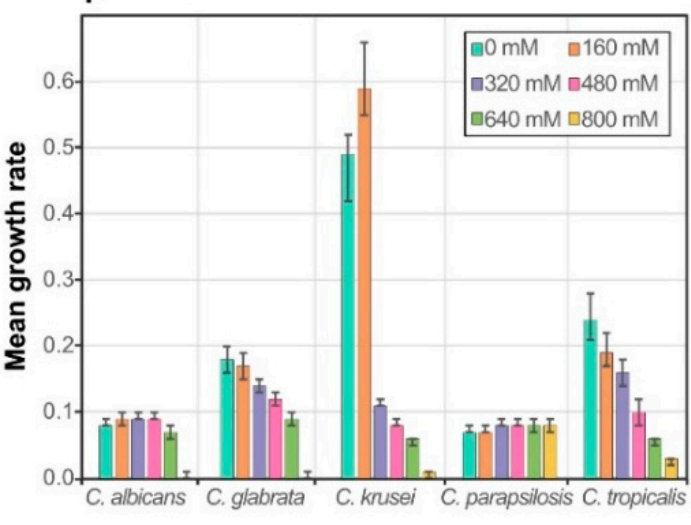

Figure 4. Lactic acid tolerance of Candida species using glycerol as carbon source Growth response to lactic acid stress with $2 \%$ Glycerol as carbon source; (A) C. albicans; (B) C. glabrata; (C) C. krusei; (D) C. parapsilosis; (E) C. tropicalis; (F) Mean growth rates of different Candida species to lactic acid stress, error bars represent confidence interval of 95\%; Each data point represents mean growth rate of triplicates measurements; Asterisks represents statistical difference of one condition to the next lower lactic acid concentration $\left({ }^{*} p \leq 0.001\right)$. Raw data can be found in Supplementary Table S4.

\section{Discussion}

Lactic acid and low $\mathrm{pH}$ are considered to represent important defense mechanisms against bacterial and fungal infections in the vaginal tract [15,32]. However, little is known about the effective range of 
lactic acid at low $\mathrm{pH}$ on Candida species usually connected to humans. In this study, we evaluated the tolerance to lactic acid of five relevant Candida species (C. albicans, C. glabrata, C. krusei, C. parapsilosis, and $C$. tropicalis). In order to analyze the variation between isolates we analyzed populations of isolates collected from the Lower Austria and Vienna region over several years. Our data were generated in a highly standardized manner using automated inoculations and continuous measurements of growth parameters of the treated isolates. Using these methods, we define a characteristic phenotypic space for each Candida species interrogated.

Previous studies reported, that physiological concentrations $(110 \mathrm{mM})$ of lactic acid had no effect on C. albicans and C. glabrata growth [23]. We found a significant decrease in growth rate at the lowest tested concentration $(160 \mathrm{mM})$ but no evidence of a fungicidal effect in our quantitative growth analysis against any isolate ( $\sim 300$ strains) of the tested species at lactic acid concentration up to $480 \mathrm{mM}$. The epithelium in the vaginal tract is reported to be layered with Lactobacillus spp, which is thought to prevent pathogens to adhere to the epithelial cell wall [33]. It is unclear, if lactic acid reaches higher concentrations in close proximity to these Lactobacillus spp. biofilms covering the vaginal tract. The increased viscosity due to cervical mucus could decrease diffusion of lactic acid and favor establishment of micro milieus with higher concentrations. Vaginal Lactobacillus spp. were reported to grow in lactic acid concentrations of $1000 \mathrm{mM}$ at pH 4.5 [24], showing that high concentrations are not harmful to lactobacilli. Our results show that a mean reduction of growth rate by $50 \%$ (MIC50) of C. albicans and C. krusei isolates required more than $450 \mathrm{mM}$ lactic acid. For C. krusei, C. parapsilosis, and C. tropicalis higher concentrations were necessary to achieve $50 \%$ growth reduction. C. parapsilosis was the most resistant, as average growth rate was not reduced by $50 \%$ with $800 \mathrm{mM}$ lactic acid. A slightly higher MIC50 compared to our results for lactic acid has been reported for strains of C. albicans, C. glabrata and C. parapsilosis [21]. However, the MIC50 values by Cottier et al. [21] are in the upper range of our results, indicating the importance of analyzing isolate populations to detect and characterize the variability of phenotypes and responses.

In support of this, we found a high variability of lactic acid tolerance in different Candida populations. Whereas C. glabrata formed clusters of fast growing and slow growing isolates, C. krusei and $C$. tropicalis isolates growing slower at low lactic acid stress tended to be fast growing at higher concentrations. For C. glabrata isolates phenotypic variations were already reported in acetic acid response [34], adhesion, and antifungal susceptibility [35]. These isolates were retrieved from different sites (e.g., bloodstream, vaginal tract, and respiratory tract) collected from patients during diagnostic routine. Therefore, the phenotypic diversity could be explained by selective pressures in the host and differences in the genetic background of the isolates. The genetic plasticity of some Candida species is substantial. For example genetic variations in clonal populations of $C$. glabrata have been suggested to to resulte from selection processes in the human body [36]. The source of the variation could also be an intrinsic property of the species and originating from epigenetic fluctuations.

Candida species growing on glycerol as sole carbon source were found to possess an increased stress tolerance to lactic acid (Figure 4). Growth on alternative carbon sources was reported to induce tolerance mechanisms to various stress types in C. albicans. [37-39]. This is accompanied by physiological changes such as changes in the cell wall composition [40-42], which are linked to increased stress tolerance. The vaginal environment consists of approximately $50 \%$ D-lactic acid, if dominated by L. crispatus [19]. In this study, we used only L-lactic acid. No evidence was reported yet that isomers exhibit a different growth effect on Candida species. In C. alibicans L- as well as D-lactic acid is taken up by the transporter JEN1 [43]. C. albicans, C. parapsilosis, and C. glabrata possess orthologs for DLD1, a D-lactate hydrogenase in Saccharomyces cerevisiae [44]. This hints that they are able to utilize D-lactate, as well as L-lactate. Taken together, this makes it unlikely that different isomers have a different effect.

For Candida species the assimilation of different carbon sources is linked to their ability to thrive in several host niches (reviewed in Alves et al. [45]). Human $\alpha$-amylase present in Lower-Genital-Tract mucosal fluid processes glycogen to support vaginal colonization by Lactobacillus. The maltose 
and maltotriose produced by $\alpha$-amylase can be fermented by $C$. albicans but not by the other tested species [46]. In C. albicans lactate uptake is facilitated by JEN transporters [43] and used in gluconeogenesis in order to generate hexose and pentose sugars which are needed for nucleotide and cell wall synthesis [47]. In C. glabrata L-lactate dehydrogenase Cyb2 is responsible for lactate assimilation in hypoxic conditions like vaginal or gastrointestinal tract [48]. Growth of C. albicans and C. glabrata, on lactate as carbon source is slower compared to glucose $[37,38]$. Our results confirmed that also quantitatively. In addition, $C$. tropicalis and C. parapsilosis also grew significantly slower on lactate compared to their growth on glucose. In contrast, $C$. krusei reached higher growth rates on lactate than on glucose (Figure 2C) and showed increased growth rates at low amounts of lactic acid on glucose (Figure 1C), as well as on glycerol (Figure 4C). Therefore, C. krusei can in vitro utilize lactate as carbon source in presence of glucose or glycerin. Thus, it possesses a unique way to more efficiently utilize lactate compared to other Candida species. Our results also show that $C$. krusei has a high tolerance against low amounts of lactic acid. In support of our view, intracellular $\mathrm{pH}$ of $C$. krusei only slightly changes when challenged with $106 \mathrm{mM}$ lactic acid undissociated at $\mathrm{pH} 2.5$ [49]. C.krusei is a rare cause of refractory vaginitis, but harder to treat than more common C. albicans infections as it possesses an intrinsic resistance to fluconazole, a very common antifungal used against vaginal Candida infections $[14,50]$.

To fully understand the lactic acid tolerance of Candida in the vaginal tract further studies are needed, which take the human microbiome and host interactions into account. However, our results hint that the usual concentration of lactic acid in the vaginal environment is low enough to be used as carbon source by the tested Candida species and only has minor part in general defense except for local higher concentrations in the vicinity of lactobacillus biofilms. Thus, lactobacilli perhaps do not generally eliminate Candida but merely restrict local growth of them.

In conclusion, we are the first to quantitatively describe L-lactic acid tolerance in different Candida species. We show that each Candida species not only has a distinct growth response to lactic acid stress, but they also vary greatly in the populations characteristic. Furthermore, we report that lactic acid tolerance is dependent on carbon source in vitro, as Candida species are less tolerant at $160 \mathrm{mM}$ lactic acid when grown on glycerol. Lastly, our report is the first to describe C. krusei to efficiently use L-lactate as carbon source. We believe that our results contribute to the mechanisms behind the role of lactic acid.

Supplementary Materials: The following are available online at http://www.mdpi.com/2309-608X/6/4/348/s1, Table S1: List of all used strains for this study. Raw data for graph generation-Figure 1 (Table S2), Figure 3 (Table S3), Figure 4 (Table S4), Figure S1: Growth behavior of diverse Candida isolates on 2\% Lactate or 2\% Glucose as sole carbon source at $\mathrm{pH} 4$.

Author Contributions: Conceptualization, C.S.; methodology, I.Z.; software, R.B.; validation, C.S. and J.S.; formal analysis, I.Z.; investigation, I.Z.; resources, C.S., B.W. and I.-J.P.; data curation, I.Z.; writing-original draft preparation, I.Z.; writing - review and editing, all authors; visualization, I.Z.; supervision, C.S.; project administration, C.A. and C.S.; funding acquisition, C.A. and C.S. All authors have read and agreed to the published version of the manuscript.

Funding: The work was supported by a grant of the Niederösterreichische Forschungs- und Bildungsges.m.b.H. LSC16-016 to C.S. and C.A. The Bioactive Microbial Metabolites facility (BiMM) is supported by a grant of the Niederösterreichische Forschungs- und Bildungsges.m.b.H (NfB) (K3-G-2/026-2013).

Acknowledgments: We would like to thank Krishna Vasant Mutanwad for critical reading of the manuscript and Desislava Yankova for excellent technical assistance.

Conflicts of Interest: The authors declare no conflict of interest.

\section{References}

1. Gilbert, J.A.; Blaser, M.J.; Caporaso, J.G.; Jansson, J.K.; Lynch, S.V.; Knight, R. Current understanding of the human microbiome. Nat. Med. 2018, 24, 392-400. [CrossRef]

2. Kapitan, M.; Niemiec, M.J.; Steimle, A.; Frick, J.S.; Jacobsen, I.D. Fungi as part of the microbiota and interactions with intestinal Bacteria. Curr. Top. Microbiol. Immunol. 2019, 422, 265-301. 
3. Bart Jan Kullberg, M.C.A. Invasive Candidiasis. N. Engl. J. Med. 2015, 1445-1456. [CrossRef] [PubMed]

4. Underhill, D.M.; Iliev, I.D. The mycobiota: Interactions between commensal fungi and the host immune system. Nat. Rev. Immunol. 2014, 14, 405-416. [CrossRef] [PubMed]

5. Hall, R.A.; Noverr, M.C. Fungal interactions with the human host: Exploring the spectrum of symbiosis. Curr. Opin. Microbiol. 2017, 40, 58-64. [CrossRef] [PubMed]

6. Sustr, V.; Foessleitner, P.; Kiss, H.; Farr, A. Vulvovaginal candidosis: Current concepts, challenges and perspectives. J. Fungi 2020, 6, 267. [CrossRef]

7. Perlroth, J.; Choi, B.; Spellberg, B. Nosocomial fungal infections: Epidemiology, diagnosis, and treatment. Med. Mycol. 2007, 45, 321-346. [CrossRef]

8. Brunke, S.; Hube, B. Two unlike cousins: Candida albicans and C. glabrata infection strategies. Cell. Microbiol. 2013, 15, 701-708. [CrossRef]

9. Ahmad, K.M.; Kokošar, J.; Guo, X.; Gu, Z.; Ishchuk, O.P.; Piškur, J. Genome structure and dynamics of the yeast pathogen Candida glabrata. FEMS Yeast Res. 2014, 14, 529-535. [CrossRef]

10. Silva, S.; Negri, M.; Henriques, M.; Oliveira, R.; Williams, D.W.; Azeredo, J. Candida glabrata, Candida parapsilosis and Candida tropicalis: Biology, epidemiology, pathogenicity and antifungal resistance. FEMS Microbiol. Rev. 2012, 36, 288-305. [CrossRef]

11. Turner, S.A.; Butler, G. The Candida Pathogenic Species Complex. Cold Spring Harb. Perspect. Med. 2014, 4, a019778. [CrossRef] [PubMed]

12. Rhodes, J.; Fisher, M.C. Global epidemiology of emerging Candida auris. Curr. Opin. Microbiol. 2019, 52, 84-89. [CrossRef] [PubMed]

13. Pande, K.; Chen, C.; Noble, S.M. Passage through the mammalian gut triggers a phenotypic switch that promotes Candida albicans commensalism. Nat. Genet. 2013, 45, 1088-1091. [CrossRef] [PubMed]

14. Sobel, J.D. Vulvovaginal candidosis. Lancet 2007, 369, 1961-1971. [CrossRef]

15. O'Hanlon, D.E.; Moench, T.R.; Cone, R.A. Vaginal $\mathrm{pH}$ and microbicidal lactic acid when lactobacilli dominate the microbiota. PLoS ONE 2013, 8, e80074. [CrossRef] [PubMed]

16. Van De Wijgert, J.H.H.M.; Borgdorff, H.; Verhelst, R.; Crucitti, T.; Francis, S.; Verstraelen, H.; Jespers, V. The vaginal microbiota: What have we learned after a decade of molecular characterization? PLoS ONE 2014, 9, e105998. [CrossRef] [PubMed]

17. Lebeer, S.; Vanderleyden, J.; De Keersmaecker, S.C.J. Genes and molecules of lactobacilli supporting probiotic action. Microbiol. Mol. Biol. Rev. 2008, 72, 728-764. [CrossRef]

18. Boskey, E.R.; Cone, R.A.; Whaley, K.J.; Moench, T.R. Origins of vaginal acidity: High D/L lactate ratio is consistent with bacteria being the primary source. Hum. Reprod. 2001, 16, 1809-1813. [CrossRef]

19. Witkin, S.S.; Mendes-Soares, H.; Linhares, I.M.; Jayaram, A.; Ledger, W.J.; Forney, L.J. Influence of vaginal bacteria and D- and L-lactic acid isomers on vaginal extracellular matrix metalloproteinase inducer: Implications for protection against upper genital tract infections. MBio 2013. [CrossRef]

20. Beyer, R.; Jandric, Z.; Zutz, C.; Gregori, C.; Willinger, B.; Jacobsen, I.D.; Kovarik, P.; Strauss, J.; Schüller, C. Competition of Candida glabrata against Lactobacillus is Hog1 dependent. Cell. Microbiol. 2018, 20, e12943. [CrossRef]

21. Cottier, F.; Tan, A.S.M.; Xu, X.; Wang, Y.; Pavelka, N. MIG1 regulates resistance of Candida albicans against the fungistatic effect of weak organic acids. Eukaryot. Cell 2015, 14, 1054-1061. [CrossRef] [PubMed]

22. Kohler, G.A.; Assefa, S.; Reid, G. Probiotic interference of lactobacillus rhamnosus GR-1 and lactobacillus reuteri RC-14 with the opportunistic fungal pathogen Candida albicans. Infect. Dis. Obstet. Gynecol. 2012. [CrossRef] [PubMed]

23. Lourenço, A.; Pedro, N.A.; Salazar, S.B.; Mira, N.P. Effect of acetic acid and lactic acid at low pH in growth and azole resistance of Candida albicans and Candida glabrata. Front. Microbiol. 2019, 9, 3265. [CrossRef] [PubMed]

24. O'Hanlon, D.E.; Moench, T.R.; Cone, R.A. In vaginal fluid, bacteria associated with bacterial vaginosis can be suppressed with lactic acid but not hydrogen peroxide. BMC Infect. Dis. 2011, 11, 200. [CrossRef] [PubMed]

25. Cormack, B.P.; Falkow, S. Efficient homologous and illegitimate recombination in the opportunistic yeast pathogen Candida glabrata. Genetics 1999, 151, 979-987. [PubMed]

26. Sprouffske, K.; Wagner, A. Growthcurver: An R package for obtaining interpretable metrics from microbial growth curves. BMC Bioinform. 2016, 17, 172. [CrossRef] 
27. R Core Team R: A Language and Environment for Statistical Computing. Available online: https://www.rproject.org/ (accessed on 5 October 2020).

28. Patil, I. ggstatsplot: "ggplot2" Based Plots with Statistical Details. Available online: https://cran.r-project. org/package=ggstatsplot (accessed on 15 October 2020).

29. Wickham, H. ggplot2 Elegant Graphics for Data Analysis; Springer: Berlin/Heidelberg, Germany, 2016; ISBN 9780387981406.

30. Willems, H.M.E.; Ahmed, S.S.; Liu, J.; Xu, Z.; Peters, B.M. Vulvovaginal Candidiasis: A Current Understanding and Burning Questions. J. Fungi 2020, 6, 27. [CrossRef]

31. Owen, D.H.; Katz, D.F. A vaginal fluid simulant. Contraception 1999, 59, 91-95. [CrossRef]

32. Ravel, J.; Gajer, P.; Abdo, Z.; Schneider, G.M.; Koenig, S.S.K.; McCulle, S.L.; Karlebach, S.; Gorle, R.; Russell, J.; Tacket, C.O.; et al. Vaginal microbiome of reproductive-age women. Proc. Natl. Acad. Sci. USA 2011, 108, 4680-4687. [CrossRef]

33. Osset, J.; Bartolomé, R.M.; García, E.; Andreu, A. Assessment of the capacity of Lactobacillus to inhibit the growth of uropathogens and block their adhesion to vaginal epithelial cells. J. Infect. Dis. 2001, 183, 485-491. [CrossRef]

34. Cunha, D.V.; Salazar, S.B.; Lopes, M.M.; Mira, N.P. Mechanistic Insights Underlying Tolerance to Acetic Acid Stress in Vaginal Candida glabrata Clinical Isolates. Front. Microbiol. 2017, 8, 259. [CrossRef] [PubMed]

35. Jong, H.S.; Myung, J.C.; Jeong, W.S.; Jung, S.I.; Cho, D.; Seung, J.K.; Soo, H.K.; Myung, G.S.; Soon, P.S.; Dong, W.R. Changes in karyotype and azole susceptibility of sequential bloodstream isolates from patients with Candida glabrata candidemia. J. Clin. Microbiol. 2007, 45, 2385-2391. [CrossRef]

36. Carreté, L.; Ksiezopolska, E.; Gómez-Molero, E.; Angoulvant, A.; Bader, O.; Fairhead, C.; Gabaldón, T. Genome comparisons of Candida glabrata serial clinical isolates reveal patterns of genetic variation in infecting clonal populations. Front. Microbiol. 2019, 10, 112. [CrossRef] [PubMed]

37. Chew, S.Y.; Ho, K.L.; Cheah, Y.K.; Sandai, D.; Brown, A.J.P.; Lung Than, L.T. Physiologically relevant alternative carbon sources modulate biofilm formation, cell wall architecture, and the stress and antifungal resistance of Candida glabrata. Int. J. Mol. Sci. 2019, 20, 3172. [CrossRef] [PubMed]

38. Ene, I.V.; Adya, A.K.; Wehmeier, S.; Brand, A.C.; Maccallum, D.M.; Gow, N.A.R.; Brown, A.J.P. Host carbon sources modulate cell wall architecture, drug resistance and virulence in a fungal pathogen. Cell. Microbiol. 2012, 14, 1319-1335. [CrossRef]

39. Williams, R.B.; Lorenz, M.C. Multiple alternative carbon pathways combine to promote Candida albicans stress resistance, immune interactions, and virulence. MBio 2020. [CrossRef]

40. Ene, I.V.; Heilmann, C.J.; Sorgo, A.G.; Walker, L.A.; De Koster, C.G.; Munro, C.A.; Klis, F.M.; Brown, A.J.P. Carbon source-induced reprogramming of the cell wall proteome and secretome modulates the adherence and drug resistance of the fungal pathogen Candida albicans. Proteomics 2012, 12, 3164-3179. [CrossRef]

41. Ene, I.V.; Cheng, S.C.; Netea, M.G.; Brown, A.J.P. Growth of Candida albicans cells on the physiologically relevant carbon source lactate affects their recognition and phagocytosis by immune cells. Infect. Immun. 2013, 81, 238-248. [CrossRef]

42. Ene, I.V.; Walker, L.A.; Schiavone, M.; Lee, K.K.; Martin-Yken, H.; Dague, E.; Gow, N.A.R.; Munro, C.A.; Brown, A.J.P. Cell wall remodeling enzymes modulate fungal cell wall elasticity and osmotic stress resistance. MBio 2015. [CrossRef]

43. Soares-Silva, I.; Paiva, S.; Kötter, P.; Entian, K.D.; Casal, M. The disruption of JEN1 from Candida albicans impairs the transport of lactate. Mol. Membr. Biol. 2004, 21, 403-411. [CrossRef]

44. Lodi, T.; Ferrero, I. Isolation of the DLD gene of Saccharomyces cerevisiae encoding the mitochondrial enzyme D-lactate ferricytochrome c oxidoreductase. MGG Mol. Gen. Genet. 1993, 238, 315-324. [CrossRef] [PubMed]

45. Alves, R.; Barata-Antunes, C.; Casal, M.; Brown, A.J.P.; van Dijck, P.; Paiva, S. Adapting to survive: How Candida overcomes host-imposed constraints during human colonization. PLoS Pathog. 2020, 16, e1008478. [CrossRef] [PubMed]

46. Spear, G.T.; French, A.L.; Gilbert, D.; Zariffard, M.R.; Mirmonsef, P.; Sullivan, T.H.; Spear, W.W.; Landay, A.; Micci, S.; Lee, B.H.; et al. Human $\alpha$-amylase present in lower-genital-tract mucosal fluid processes glycogen to support vaginal colonization by Lactobacillus. J. Infect. Dis. 2014, 210, 1019-1028. [CrossRef] [PubMed]

47. Turcotte, B.; Liang, X.B.; Robert, F.; Soontorngun, N. Transcriptional regulation of nonfermentable carbon utilization in budding yeast. FEMS Yeast Res. 2010, 10, 2-13. [CrossRef] [PubMed] 
48. Ueno, K.; Matsumoto, Y.; Uno, J.; Sasamoto, K.; Sekimizu, K.; Kinjo, Y.; Chibana, H. Intestinal resident yeast Candida glabrata requires Cyb2p-Mediated lactate assimilation to adapt in mouse intestine. PLoS ONE 2011, 6, e24759. [CrossRef] [PubMed]

49. Halm, M.; Hornbæk, T.; Arneborg, N.; Sefa-Dedeh, S.; Jespersen, L. Lactic acid tolerance determined by measurement of intracellular $\mathrm{pH}$ of single cells of Candida krusei and Saccharomyces cerevisiae isolated from fermented maize dough. Int. J. Food Microbiol. 2004, 94, 97-103. [CrossRef]

50. Singh, S.; Sobel, J.D.; Bhargava, P.; Boikov, D.; Vazquez, J.A. Vaginitis due to Candida krusei: Epidemiology, clinical aspects, and therapy. Clin. Infect. Dis. 2002, 35, 1066-1070. [CrossRef]

Publisher's Note: MDPI stays neutral with regard to jurisdictional claims in published maps and institutional affiliations.

(C) 2020 by the authors. Licensee MDPI, Basel, Switzerland. This article is an open access article distributed under the terms and conditions of the Creative Commons Attribution (CC BY) license (http://creativecommons.org/licenses/by/4.0/). 